\title{
ASYMPTOTIC BEHAVIOUR OF SEMIGROUPS OF OPERATORS
}

\author{
C. J. K. BATTY
}

St. John's College, Oxford OX1 3JP, England

1. Introduction. This article is a survey of results obtained since 1986 concerning the relationship between spectral properties of semigroups of operators and their asymptotic behaviour. Amongst the prototypes were results of Sklyar and Shirman [36], and of Katznelson and Tzafriri [23]. The central result, obtained independently and simultaneously, by Lyubich and Phóng [28] and by Arendt and the author [6], is that a bounded one-parameter semigroup of operators converges to zero in the strong operator topology if the peripheral spectrum is countable and the peripheral point spectrum of the adjoint is empty. We shall describe this result in some detail, and various subsequent developments including those of Arendt and Prüss [8], Phóng [35], [12], [13], Esterle, Strouse and Zouakia [16], [17], El Mennaoui [15], Nagel, Räbiger and Huang [31], [21], and Greenfield [19], [11], in less detail.

The term "semigroup of operators" may refer to the family $\left\{T^{n}: n=\right.$ $0,1,2, \ldots\}$ of powers of a single bounded linear operator $T$ on a Banach space $X$ (discrete one-parameter semigroup); to a $C_{0}$-semigroup $\{T(t): t \geq 0\}$ on $X$ (continuous one-parameter semigroup); or to a strongly continuous representation $T$ on $X$ of a suitable locally compact semigroup $S$ (general semigroup), for example $S=\mathbb{R}_{+}^{m}$ or $S=\mathbb{Z}_{+}^{m}$ (multi-parameter semigroup). We shall consider these cases in Sections 2, 3, and 5, respectively. The main result of Section 5 (Theorem 5.6) includes those of Sections 2 and 3, once certain natural identifications have been made, but some of the subsidiary results are sharper in the one-parameter case. Readers who are interested only in (discrete or continuous) one-parameter semigroups do not need to trouble themselves with the extra generality of Section 5, but Theorem 5.3 (a dilation theorem for isometries, due to Greenfield) may be

1991 Mathematics Subject Classification: 47-02, 47A05, 47D03.

The paper is in final form and no version of it will be published elsewhere. 
of interest. In Section 4, we describe a variety of other extensions of the central result in the one-parameter setting.

As will become apparent (see Proposition 2.6), the study of semigroups of isometries is essentially the complement of that of semigroups which converge to zero. There are certain spectral properties of general isometric semigroups which are rather elementary in the one-parameter cases, but less so for multi-parameter and general semigroups. These will be described in Sections 5 and 6 .

Throughout, $X$ will be a complex Banach space, and $\mathcal{B}(X)$ will be the space of bounded linear operators on $X$. Operator-valued integrals will be convergent in the strong operator topology. For a linear operator $A: D(A) \rightarrow X$, with domain $D(A)$ in $X, \varrho(A), \sigma(A), P \sigma(A)$, and $A \sigma(A)$ will denote the resolvent set, spectrum, point spectrum, and approximate point spectrum of $A$, respectively; if $D(A)$ is dense in $X, P \sigma\left(A^{*}\right)$ will denote the point spectrum of the adjoint $A^{*}$ of $A$. We shall denote the kernel and range of $A$ by $\operatorname{Ker} A$ and $\operatorname{Ran} A$, respectively.

We shall also use the following notation:

$$
\begin{gathered}
\mathbb{Z}_{+}=\{0,1,2, \ldots\}, \quad \mathbb{R}_{+}=[0, \infty), \quad \mathbb{C}_{-}=\{\lambda \in \mathbb{C}: \operatorname{Re} \lambda \leq 0\}, \\
D=\{\lambda \in \mathbb{C}:|\lambda| \leq 1\}, \quad \Gamma=\{\lambda \in \mathbb{C}:|\lambda|=1\} .
\end{gathered}
$$

2. Discrete one-parameter semigroups. In this section, we consider a semigroup $\left\{T^{n}: n \in \mathbb{Z}_{+}\right\}$, where $T$ is a bounded linear operator on a complex Banach space. We shall assume that $T$ is power-bounded, so that $\sup _{n \in \mathbb{Z}_{+}}\left\|T^{n}\right\|<\infty$. It follows that $\sigma(T)$ is contained in the closed unit disc $D$. We shall be interested only in the peripheral (unitary) part of the spectrum $\sigma(T) \cap \Gamma$, where $\Gamma$ is the unit circle.

The following is the general operator-theoretic result obtained by Katznelson and Tzafriri.

TheOREM 2.1 [23, Theorem 5]. Let $T$ be a power-bounded operator on $X$, and $\left(a_{n}\right)$ be a sequence in $\ell^{1}\left(\mathbb{Z}_{+}\right)$, and suppose that the power series $g(z)=\sum a_{n} z^{n}$ is of spectral synthesis with respect to $\sigma(T) \cap \Gamma$. Then $\left\|T^{n} g(T)\right\| \rightarrow 0$ as $n \rightarrow \infty$.

The following corollary, obtained by taking $g(z)=1-z$, is the case to which most attention has been given, both in [23] and by subsequent authors.

Corollary 2.2 [23, Theorem 1]. Let $T$ be a power-bounded operator on $X$ such that $\sigma(T) \cap \Gamma \subseteq\{1\}$. Then $\left\|T^{n}-T^{n+1}\right\| \rightarrow 0$ as $n \rightarrow \infty\left(^{1}\right)$.

The proof of Corollary 2.2 in [23] used harmonic analysis to establish a Tauberian theorem for power series, from which Corollary 2.2 follows easily. Methods of this type will not be discussed in this article.

A simple consequence of Corollary 2.2 is the classical result of Gelfand [18] that an operator $T$ with $\sigma(T)=\{1\}$ and $\sup _{n \in \mathbb{Z}}\left\|T^{n}\right\|<\infty$ is the identity. Allan

$\left({ }^{1}\right)$ Editorial note: See also pp. $372-373$ in this volume. 
and Ransford [3] have shown that Corollary 2.2 may be deduced from Gelfand's Theorem by a suitable construction.

Another corollary of Theorem 2.1 is the following. Here, we write $T^{n} \stackrel{\mathrm{s}}{\rightarrow} 0$ to indicate that $T^{n} \rightarrow 0$ in the strong operator topology, that is, $\left\|T^{n} x\right\| \rightarrow 0$ for each $x$ in $X$.

Corollary 2.3. Let $T$ be a power-bounded operator on $X$ such that $\sigma(T) \cap \Gamma$ is finite and $P \sigma\left(T^{*}\right) \cap \Gamma$ is empty. Then $T^{n} \stackrel{\mathrm{s}}{\rightarrow} 0$ as $n \rightarrow \infty$.

Proof. Let $g(z)$ be the polynomial

$$
g(z)=\prod_{\lambda \in \sigma(T) \cap \Gamma}(z-\lambda) .
$$

Since $g$ vanishes on the finite set $\sigma(T) \cap \Gamma$, it is automatically of spectral synthesis, so Theorem 2.1 implies that $\left\|T^{n} g(T)\right\| \rightarrow 0$. Since $P \sigma\left(T^{*}\right) \cap \Gamma$ is empty, $T-\lambda I$ has dense range for each $\lambda$ in $\sigma(T) \Gamma \Gamma$, so $g(T)$ has dense range. An easy approximation argument completes the proof.

The central result of this section, Theorem 2.5, is an improvement of Corollary 2.3. In order to appreciate the sharpness of Theorem 2.5, it is instructive to consider the example of multipliers.

ExAmple 2.4. (1) Let $X=L^{p}(\Omega, \mu)$, where $(\Omega, \mu)$ is a $\sigma$-finite measure space and $1 \leq p<\infty$. Let $h: \Omega \rightarrow D$ be a measurable function, and $T$ be the multiplier on $X$ defined by $T f=h f$, so $\|T\| \leq 1$. Then $\sigma(T)$ is the support of the measure $\nu$ induced on $D$ by $\mu$ via $h, \nu(E)=\mu\left(h^{-1}(E)\right)$, and

$$
P \sigma(T)=P \sigma\left(T^{*}\right)=\{\lambda \in D: \nu\{\lambda\}>0\}=\{\text { atoms of } \nu\} .
$$

Suppose that $\nu$ has an atom $\lambda$ in $\Gamma$. Then there is a measurable subset $E$ of $h^{-1}\{\lambda\}$ such that $0<\mu(E)<\infty$. If $f$ is the characteristic function of $E$, then $T^{n} f=\lambda^{n} f$, so $\left\|T^{n} f\right\|$ does not tend to 0 .

On the other hand, suppose that $\nu(\Gamma)=0$. Let

$Y=\{g \in X$ : there exists $\delta>0$ such that $g(\omega)=0$ whenever $|h(\omega)|>1-\delta\}$.

For $g$ in $Y,\left\|T^{n} g\right\| \leq(1-\delta)^{n}\|g\| \rightarrow 0$. The assumption that $\nu(\Gamma)=0$ implies that $Y$ is dense in $X$, and it follows that $T^{n} \stackrel{\mathrm{s}}{\rightarrow} 0$.

In particular, if $\sigma(T) \cap \Gamma$ is countable and $P \sigma\left(T^{*}\right) \cap \Gamma$ is empty, then $(\operatorname{supp} \nu)$ $\cap \Gamma$ is countable and $\nu$ has no atoms in $\Gamma$, so $\nu(\Gamma)=0$, and $T^{n} \stackrel{\mathrm{s}}{\rightarrow} 0$.

(2) Let $\Omega$ be a closed uncountable subset of $\Gamma$. There is a non-atomic probability measure $\mu$ on $\Omega$. Let $X=L^{p}(\Omega, \mu)$, where $1 \leq p<\infty$, and $T$ be the multiplier on $X:(T f)(z)=z f(z)$. Then $\sigma(T) \subseteq \Omega, P \sigma\left(T^{*}\right)$ is empty, but $T$ is isometric.

Suppose that $T$ is an operator such that $T^{n} \stackrel{\mathrm{s}}{\rightarrow} 0$. Then $T$ is power-bounded (by the Uniform Boundedness Principle), and $P \sigma(T) \cap \Gamma$ and $P \sigma\left(T^{*}\right) \cap \Gamma$ are 
empty. It is also not difficult to see that, for any power-bounded operator, $P \sigma(T) \cap$ $\Gamma \subseteq P \sigma\left(T^{*}\right) \cap \Gamma$. These observations, together with Example 2.4, show that the assumptions in the following theorem are natural.

TheOREM 2.5 [5, Theorem 5.1]. Let $T$ be a power-bounded operator on $X$ such that $\sigma(T) \cap \Gamma$ is countable and $P \sigma\left(T^{*}\right) \cap \Gamma$ is empty. Then $T^{n} \stackrel{\mathrm{s}}{\rightarrow} 0$ as $n \rightarrow \infty$.

Theorem 2.5 was proved in [5] by a method based on an estimate for power series due to Allan, O'Farrell and Ransford [2] who were concerned to generalise the Tauberian theorem of Katznelson and Tzafriri [23, Theorem 2] and to exhibit analogies with Tauberian theorems arising in modern proofs of the Prime Number Theorem [33], [24]. In addition, the proof in [5] used a (possibly transfinite) inductive argument based on the successive derived sets of $\sigma(T) \cap \Gamma$.

It is also possible to prove Theorem 2.5 by modifying the functional-analytic proof given in [28] of the continuous version of Theorem 2.5 (see Theorem 3.1). That proof was obtained independently of [5], and the discrete version will be described below.

Subsequently, a third proof of Theorem 2.5 has been given in [16]. There, harmonic analysis is used to establish, under the conditions of Theorem 2.5, that the span of $\bigcup \operatorname{Ran} g(T)$ is dense in $X$, where the union is taken over all power series $g(z)$, with absolutely summable coefficients, which vanish on the countable set $\sigma(T) \cap \Gamma$. Theorem 2.5 then follows from Theorem 2.1.

The proof in [28] depends on the following construction.

Proposition 2.6. Let $T$ be a power-bounded operator on $X$. There is a $B a$ nach space $E$, a bounded linear map $Q$ of $X$ into $E$ with dense range, and an isometry $U$ on $E$ such that

(1) If $x \in X$ and $Q x=0$, then $\left\|T^{n} x\right\| \rightarrow 0$;

(2) $Q T=U Q$

(3) $\sigma(U) \subseteq \sigma(T), P \sigma\left(U^{*}\right) \subseteq P \sigma\left(T^{*}\right)$.

Proof. Define a seminorm $\ell$ on $X$ by

$$
\ell(x)=\limsup _{n \rightarrow \infty}\left\|T^{n} x\right\| \quad(x \in X) .
$$

Let $L=\{x \in X: \ell(x)=0\}, \tilde{\ell}$ be the associated norm on $X / L$, and $E$ be the completion of $(X / L, \widetilde{\ell})$. Let $Q: X \rightarrow E$ be the natural quotient map. Then $Q$ has dense range, (1) holds, and $T$ induces an isometry $U$ on $E$ satisfying (2).

Let $\lambda \in \varrho(T)$, and $R_{\lambda}=(\lambda I-T)^{-1}$. Since $R_{\lambda}$ commutes with $T, R_{\lambda}$ induces an operator $S_{\lambda}$ on $E$ such that $S_{\lambda}(\lambda I-U)=I=(\lambda I-U) S_{\lambda}$, so $\lambda \in \varrho(U)$. Thus $\sigma(U) \subseteq \sigma(T)$. If $\lambda \in P \sigma\left(U^{*}\right)$, then $U^{*} \psi=\lambda \psi$ for some non-zero $\psi$ in $E^{*}$, so $T^{*}\left(Q^{*} \psi\right)=\lambda Q^{*} \psi$ and $Q^{*} \psi \neq 0$. Thus $\lambda \in P \sigma\left(T^{*}\right)$.

Proof of Theorem 2.5 (see [28]). Let $E, Q$ and $U$ be as in Proposition 2.6. Since $U$ is isometric, $A \sigma(U) \subseteq \Gamma$, so the topological boundary $\partial \sigma(U)$ of $\sigma(U)$ 
is contained in $\Gamma$. Hence $\partial \sigma(U) \subseteq \sigma(T) \cap \Gamma \neq \Gamma$. It follows that $\sigma(U) \subseteq \Gamma$, so $U$ is invertible with countable spectrum.

Suppose, by way of contradiction, that $E \neq\{0\}$. Then $\sigma(U)$ is non-empty and countable, and therefore has an isolated point $\lambda$, which must be an eigenvalue of $U$. Moreover, there is a bounded projection $P$ of $E$ onto the $\lambda$-eigenspace $E_{\lambda}$ of $U$. Choose a non-zero functional $\psi$ in $\left(E_{\lambda}\right)^{*}$. Then $U^{*}\left(P^{*} \psi\right)=\lambda P^{*} \psi$, so $\lambda \in P \sigma\left(U^{*}\right) \cap \Gamma \subseteq P \sigma\left(T^{*}\right) \cap \Gamma$. This contradicts the assumption. Hence $E=\{0\}$, so $T^{n} \stackrel{\mathrm{s}}{\rightarrow} 0$, by Proposition 2.6(1).

Remark 2.7. 1. Note that the first part of the proof above shows that if $U$ is an isometry, then either $\sigma(U)=D$ or $U$ is invertible and $\sigma(U) \subseteq \Gamma$.

2. Phóng [35] has shown how Theorem 2.1 may be deduced from Proposition 2.6. In the context of Theorem 2.1, we may assume that $\Gamma$ is not contained in $\sigma(T)$ and therefore not in $\sigma(U)$. It follows from the previous remark that $U$ is invertible. If $g$ is of spectral synthesis for $\sigma(T) \cap \Gamma$ and hence for $\sigma(U)$, then $g(U)=0$. Hence $Q g(T) x=g(U) Q x=0$ for all $x$ in $X$, so $T^{n} g(T) \stackrel{\mathrm{s}}{\rightarrow} 0$. Now, one applies this result with $X$ replaced by $\mathcal{B}(X)$, and $T$ replaced by $\widetilde{T}$, where $\widetilde{T}(V)=T V$, to deduce that $\left\|T^{n} g(T) V\right\| \rightarrow 0$ for all $V$ in $\mathcal{B}(X)$. Taking $V=I$ gives the result.

3. There is a result for operators on a Hilbert space, due to Sz.-Nagy and Foiaş [32], which is related to Theorem 2.5. They showed that if $T$ is a completely non-unitary contraction on a Hilbert space $X$ and $\sigma(T) \cap \Gamma$ is null (for Haar measure on $\Gamma$ ), then $T^{n} \stackrel{\mathrm{s}}{\rightarrow} 0$. Example 2.4(2) shows that it is not possible to replace the assumption of complete non-unitarity by the weaker condition that $P \sigma\left(T^{*}\right) \cap \Gamma$ is empty, even when $X$ is a Hilbert space.

4. There are operators $T$ on the Hilbert space $\ell^{2}$ such that $\left\|T^{n}\right\|=O(n)$ as $n \rightarrow \infty, \sigma(T) \cap \Gamma=\{1\}, 1 \notin P \sigma(T) \cup P \sigma\left(T^{*}\right)$, but $T^{n}$ does not converge to 0 strongly. Such an operator may be defined by

$$
(T x)_{2 m-1}=\left(\frac{m-1}{m}\right)\left(x_{2 m-1}+x_{2 m}\right), \quad(T x)_{2 m}=\left(\frac{m-1}{m}\right) x_{2 m}
$$

(see [4, Example 2.5], [12, Example 6]) $\left(^{2}\right)$.

3. Continuous one-parameter semigroups. In this section, we consider a $C_{0}$-semigroup $T=\left\{T(t): t \in \mathbb{R}_{+}\right\}$of bounded linear operators on a complex Banach space $X$. We shall denote the generator of $T$ by $A$. We shall assume that $T$ is bounded, so that $\sup _{t \in \mathbb{R}_{+}}\|T(t)\|<\infty$ and $\sigma(A) \subseteq \mathbb{C}_{-}$.

The following result is the analogue of Theorem 2.5 , and was proved in exactly this form in [5] and [28], simultaneously and independently. The norm-continuous case, which can be deduced from Theorem 2.5, had earlier been proved in [36].

$\left(^{2}\right)$ Editorial note: See also p. 373 in this volume. 
In the strongly continuous case, the result does not follow from Theorem 2.5 because of the failure of the Spectral Mapping Theorem-in general, $\sigma(T(t)) \neq$ $\left\{e^{\lambda t}: \lambda \in \sigma(A)\right\}$. Indeed, it is not difficult to construct examples of semigroups (even multiplier semigroups on $L^{p}$-spaces) satisfying the conditions of Theorem 3.1 , where $\sigma(T(t))=D$ for all $t>0$ (see [5, Example 5.5]).

TheOREM 3.1 [5, Theorem 2.4], [28]. Let $\left\{T(t): t \in \mathbb{R}_{+}\right\}$be a bounded $C_{0^{-}}$ semigroup on $X$ with generator $A$, and suppose that $\sigma(A) \cap i \mathbb{R}$ is countable and $P \sigma\left(A^{*}\right) \cap i \mathbb{R}$ is empty. Then $T(t) \stackrel{\mathrm{s}}{\rightarrow} 0$ as $t \rightarrow \infty$.

The proof of Theorem 3.1 given in [5] is analogous to the proof of Theorem 2.5 in the same paper. In the case when $\sigma(A) \cap i \mathbb{R}$ is empty, the result can be deduced from a Tauberian theorem of Ingham [22], but we will describe here the much simpler method, devised by Korevaar [24], which uses an estimate of a contour integral which can be modified to provide the basis of the proof of the general case of the theorem. Although the operator-valued integrals are to be taken in the strong-operator topology, the estimates hold in the operator norm.

Suppose then that $\sigma(A) \cap i \mathbb{R}$ is empty. Let $R>0, \gamma_{+}$and $\gamma_{-}$be the left and right halves of the circle $|z|=R$, and $\gamma^{\prime}$ be a path in $\{z \in \varrho(A): \operatorname{Re} z<0\}$ from $i R$ to $-i R$. Let $g_{t}(z)=\int_{0}^{t} e^{-s z} T(s) d s$, so $g_{t}$ is holomorphic on $\mathbb{C}$ and

$$
e^{t z} g_{t}(z)=(z I-A)^{-1}\left(e^{t z} I-T(t)\right) \quad(z \in \varrho(A)) .
$$

By Cauchy's Theorem,

$$
\begin{aligned}
T(t) A^{-1}= & -\frac{1}{2 \pi i} \int_{\gamma_{+} \cup \gamma^{\prime}}\left(1+\frac{z^{2}}{R^{2}}\right)(z I-A)^{-1} T(t) \frac{d z}{z} \\
= & -\frac{1}{2 \pi i} \int_{\gamma_{+}}\left(1+\frac{z^{2}}{R^{2}}\right)(z I-A)^{-1} T(t) \frac{d z}{z} \\
& -\frac{1}{2 \pi i} \int_{\gamma^{\prime}}\left(1+\frac{z^{2}}{R^{2}}\right)(z I-A)^{-1} e^{t z} \frac{d z}{z} \\
& +\frac{1}{2 \pi i} \int_{\gamma_{-}}\left(1+\frac{z^{2}}{R^{2}}\right) g_{t}(z) e^{t z} \frac{d z}{z} .
\end{aligned}
$$

As $t \rightarrow \infty$, the second integral in $(*)$ tends to zero, by the Dominated Convergence Theorem. On $\gamma_{ \pm}$,

$$
\left|1+\frac{z^{2}}{R^{2}}\right|=\frac{2|\operatorname{Re} z|}{R} .
$$

On $\gamma_{+}$,

$$
\left\|(z I-A)^{-1} T(t)\right\|=\left\|\int_{0}^{\infty} e^{-s z} T(s+t) d t\right\| \leq \frac{M}{\operatorname{Re} z},
$$


where $M=\sup _{t \in \mathbb{R}_{+}}\|T(t)\|$. On $\gamma_{-}$,

$$
\left\|e^{t z} g_{t}(z)\right\|=\left\|\int_{0}^{t} e^{(t-s) z} T(s) d s\right\| \leq \frac{M}{|\operatorname{Re} z|} .
$$

Hence both the first and third integrals in $(*)$ are bounded in norm by $M / R$, so

$$
\limsup _{t \rightarrow \infty}\left\|T(t) A^{-1}\right\| \leq \frac{2 M}{R} .
$$

Letting $R \rightarrow \infty$, it follows that $\left\|T(t) A^{-1}\right\| \rightarrow 0$ as $t \rightarrow \infty$. Since $A^{-1}$ has dense range in $X$ and $T$ is bounded, it follows that $T(t) \stackrel{\mathrm{s}}{\rightarrow} 0$.

The proof of Theorem 3.1 given in [28] is an adaptation of the proof of Theorem 2.5 described in Section 2. One performs a construction analogous to Proposition 2.6 (the proof of the spectral inclusion is a little more difficult now). One also needs the fact that the generator of a $C_{0}$-semigroup of isometries on a non-zero Banach space has non-empty spectrum. This follows directly from the special case of Theorem 3.1 which we have proved above by contour integral methods. However, it may also be established by the following argument.

Proposition 3.2 [28]. Let $A$ be the generator of a $C_{0}$-semigroup $\{T(t): t \in$ $\left.\mathbb{R}_{+}\right\}$of isometries on $X$. Either $\sigma(A)=\mathbb{C}_{-}$, or $T(t)$ is invertible for each $t>0$ and $\sigma(A) \subseteq i \mathbb{R}$. In particular, if $X \neq\{0\}$, then $\sigma(A)$ is non-empty.

Proof. For $\operatorname{Re} \lambda<0$ and $x$ in $D(A)$,

$$
\begin{aligned}
e^{-(\operatorname{Re} \lambda) t}\|x\| & =\left\|e^{-\lambda t} T(t) x\right\|=\left\|x+\int_{0}^{t} e^{-\lambda s} T(s)(A-\lambda I) x d s\right\| \\
& \leq\|x\|+\frac{e^{-(\operatorname{Re} \lambda) t}-1}{-\operatorname{Re} \lambda}\|(A-\lambda I) x\| .
\end{aligned}
$$

Hence

$$
\|(A-\lambda I) x\| \geq(-\operatorname{Re} \lambda)\|x\| .
$$

This shows that $-A$ is dissipative, and that $\lambda$ is not an approximate eigenvalue of $A$. Thus $\partial \sigma(A) \subseteq i \mathbb{R}$, so $\sigma(A)=\mathbb{C}_{-}$or $\sigma(A) \subseteq i \mathbb{R}$. In the latter case, the Hille-Yosida Theorem shows that $-A$ is a generator, so each $T(t)$ is invertible.

The final statement follows from the theory of spectral subspaces for isometric (one-parameter) groups (see, for example, [27], [34, 8.1]).

A third proof of Theorem 3.1 has been given by Esterle, Strouse and Zouakia [17]. They used harmonic analysis to prove the following analogue of Theorem 2.1, which was also proved independently by Phóng [35]. Here and subsequently, we adopt the convention that the Fourier transform of a function $f$ in $L^{1}\left(\mathbb{R}_{+}\right)$is defined by

$$
\widehat{f}(\xi)=\int_{0}^{\infty} f(t) e^{i t \xi} d t
$$


and we write

$$
\widehat{f}(T)=\int_{0}^{\infty} f(t) T(t) d t .
$$

Theorem 3.3 [17, Théorème III.4], [35, Theorem 3.2]. Let $T$ be a bounded $C_{0}$-semigroup on $X$ with generator $A$, let $f \in L^{1}\left(\mathbb{R}_{+}\right)$, and suppose that $f$ is of spectral synthesis for $-i \sigma(A) \cap \mathbb{R}$. Then $\|T(t) \widehat{f}(T)\| \rightarrow 0$ as $t \rightarrow \infty$.

Rem ark 3.4. 1. It was also shown in [17] that if $\sigma(A) \cap i \mathbb{R}$ is countable and $P \sigma\left(A^{*}\right) \cap i \mathbb{R}$ is empty, then the linear span of the union of the ranges of all operators $\widehat{f}(T)$, for those $f$ in $L^{1}(\mathbb{R})$ with $\widehat{f}=0$ on $-i \sigma(A) \cap \mathbb{R}$, is dense in $X$. Theorem 3.1 then follows from Theorem 3.3.

2. Phóng [35] showed how Theorem 3.3 may be deduced from the analogue of Proposition 2.6. The argument is similar to that outlined in Remark 2.7.2, but, in order to ensure strong continuity, it is necessary to work on a subspace of $\mathcal{B}(X)$.

3. In the case when $\sigma(A) \cap i \mathbb{R}$ is empty, it was shown above, by contour integral methods, that $\left\|T(t) A^{-1}\right\| \rightarrow 0$. It is then easy to deduce that $\|T(t) \widehat{f}(T)\| \rightarrow 0$ for all $f$ in $L^{1}\left(\mathbb{R}_{+}\right)$.

4. Some extensions. In this section, we describe some extensions of Theorems 2.5 and 3.1 to other situations involving one-parameter (discrete or continuous) families of operators.

4.1. Ergodic theorems [6]. Let $T=\left\{T(t): t \in \mathbb{R}_{+}\right\}$be a (not necessarily bounded) $C_{0}$-semigroup with generator $A$. Then $T$ is said to be mean-ergodic (or Cesàro ergodic) if $P:=\lim _{t \rightarrow \infty} \frac{1}{t} \int_{0}^{t} T(s) d s$ exists in the strong operator topology; $T$ is said to be Abel ergodic if $(0, \infty) \subseteq \varrho(A)$ and $P:=\lim _{\lambda \downarrow 0} \lambda(\lambda I-A)^{-1}$ exists in the strong operator topology. Mean-ergodicity implies Abel ergodicity (and the two limits are equal) [20, Theorem 18.4.3]. For bounded semigroups, the converse holds [14, Theorem 5.1].

It is well-known [20, Theorem 18.7.3] that $T$ is Abel ergodic if and only if:

(i) $(0, \infty) \subseteq \varrho(A)$,

(ii) $\sup _{0<\lambda \leq 1}\left\|\lambda(\lambda I-A)^{-1}\right\|<\infty$,

(iii) $\operatorname{Ker} A$ separates $\operatorname{Ker} A^{*}$.

Then $P$ is a projection of $X$ onto $\operatorname{Ker} A$ with $\operatorname{Ker} P=\overline{\operatorname{Ran} A}$. Condition (iii) is equivalent to the condition that $\operatorname{Ker} A+\operatorname{Ran} A$ is dense in $X$, and it is automatically satisfied if $X$ is reflexive.

Suppose that $T$ is bounded and mean-ergodic, and that $\sigma(A) \cap i \mathbb{R}$ is countable and $P \sigma\left(A^{*}\right) \cap i \mathbb{R} \subseteq\{0\}$. Then Theorem 3.1 can be applied to the restriction of $T$ to the subspace $\overline{\operatorname{Ran} A}$ to show that $T(t) \stackrel{\mathrm{s}}{\rightarrow} P$.

The method of [5] can be applied to certain unbounded $C_{0}$-semigroups to establish mean-ergodicity [6]. The results are less elegant than Theorem 3.1; the two which follow are typical of those which have been obtained. 
TheOREM 4.1 [6, Theorem 0.2]. Let $T$ be a $C_{0}$-semigroup on $X$ with generator $A$, and suppose that $\|T(t)\|=O(t)$ as $t \rightarrow \infty, 0 \in \varrho(A)$, and $\sigma(A) \cap i \mathbb{R}$ consists only of poles of order 1 of the resolvent of $A$. Then $T$ is uniformly mean-ergodic, that is, $(1 / t) \int_{0}^{t} T(s) d s$ converges in norm as $t \rightarrow \infty$.

Theorem 4.2 [6, Theorem 2.1]. Let $T$ be a $C_{0}$-semigroup on $X$ with generator $A$, and suppose that

(1) $\|T(t)\|=O(t)$ as $t \rightarrow \infty$,

(2) $\sup _{\operatorname{Re} \lambda>0,|\lambda|<\delta}\left\|\lambda(\lambda I-A)^{-1}\right\|<\infty$ for some $\delta>0$,

(3) Ker $A$ separates $\operatorname{Ker} A^{*}$,

(4) $\sigma(A) \cap i \mathbb{R} \backslash\{0\}$ consists only of poles of order 1 of the resolvent of $A$.

Then $T$ is mean-ergodic.

Applications to triangular matrix operators, and the Cauchy problem with periodic inhomogeneity, are given in [6].

4.2. Almost periodicity [29], [11], [30]. Let $T$ be a power-bounded operator on $X$, and $\lambda \in \Gamma$. We say that $\lambda$ belongs to the (peripheral) ergodic spectrum $E \sigma(T) \cap \Gamma$ of $T$ if $\operatorname{Ker}(\lambda I-T)$ does not separate $\operatorname{Ker}\left(\lambda I-T^{*}\right)$; equivalently, if $\bar{\lambda} T$ is not (Cesàro) mean-ergodic (see [25, Section 2.1]). If $X$ is reflexive, then $E \sigma(T) \cap \Gamma$ is empty.

The operator $T$ is almost periodic if, for each $x$ in $X,\left\{T^{n} x: n \in \mathbb{Z}_{+}\right\}$is relatively compact in $X$. The splitting theorem of Jacobs, de Leeuw and Glicksberg (see [25, Theorem 4.4, p. 105]) shows that $T$ is almost periodic if and only if $X=X_{0} \oplus X_{1}$, where

$$
\begin{gathered}
X_{0}=\left\{x \in X:\left\|T^{n} x\right\| \rightarrow 0\right\}, \\
X_{1}=\overline{\operatorname{span}}\{x \in X: T x=\lambda x \text { for some } \lambda \text { in } \Gamma\} .
\end{gathered}
$$

Moreover, $T \mid X_{1}$ is invertible (and is isometric if $T$ is contractive). It is easy to deduce from this that if $T$ is almost periodic, then $E \sigma(T) \cap \Gamma$ is empty.

THEOREM 4.3 (cf. [29], [12], [30]). Suppose that $T$ is a power-bounded operator on $S$ and that $\sigma(T) \cap \Gamma$ is countable and $E \sigma(T) \cap \Gamma$ is empty. Then $T$ is almost periodic. In particular, if $X$ is reflexive and $\sigma(T) \cap \Gamma$ is countable, then $T$ is almost periodic.

There is an analogous theorem for $C_{0}$-semigroups [29], [12].

4.3. Individual theorems [12]. It was noted in [5] that if $T$ is an unbounded $C_{0}$-semigroup with generator $A$ such that $\sigma(A) \subseteq\{\lambda \in \mathbb{C}: \operatorname{Re} \lambda<0\}, x \in D(A)$, and $\sup _{t \in \mathbb{R}_{+}}\|T(t) A x\|<\infty$, then $\|T(t) x\| \rightarrow 0$ as $t \rightarrow \infty$. The argument for this is a minor adaptation of the contour integral argument given in Section 3 . By refining the argument, the following result is obtained, in which there is no assumption of boundedness, and $\sigma(A)$ may not be contained in $\mathbb{C}_{-}$. 
Theorem 4.4 [12, Theorem 1]. Let $T$ be a $C_{0}$-semigroup on $X$ with generator $A$, and suppose that $A \sigma(A) \cap i \mathbb{R}$ is empty. Let $x \in X$, and suppose that $t \mapsto T(t) x$ is uniformly continuous. Then $\|T(t) x\| \rightarrow 0$ as $t \rightarrow \infty$.

If $X$ is reflexive, $\sigma(A) \cap i \mathbb{R}$ is countable, $P \sigma(A) \cap i \mathbb{R}$ is empty, and $\sup _{t \in \mathbb{R}_{+}}\|T(t) x\|<\infty$, then $T(t) x \rightarrow 0$ weakly as $t \rightarrow \infty$ [10, Proposition 7.3], $[12$, Corollary 3].

On the other hand, an example was given in [12, Example 6] where $X=\ell^{2}, T$ is norm-continuous (so $A$ is bounded), $\|T(t)\|=O(t)$ as $t \rightarrow \infty, \sigma(A) \cap i \mathbb{R}=\{0\}$, $0 \notin P \sigma(A) \cup P \sigma\left(A^{*}\right), \sup _{t \in \mathbb{R}_{+}}\|T(t) x\|<\infty$, but $\|T(t) x\| \nrightarrow 0$.

4.4. Volterra equations [8]. Let $A$ be a closed linear operator in $X$ with dense domain $D(A)$, and let $a \in L_{\text {loc }}^{1}\left(\mathbb{R}_{+}\right)$. Let $\left\{T(t): t \in \mathbb{R}_{+}\right\}$be a bounded solution of the associated Volterra equation, so that $T(t)$ is a bounded linear operator on $X$ commuting with $A$, and

$$
T(t) x=x+A \int_{0}^{t} a(t-s) T(s) x d s \quad(x \in D(A), t>0) .
$$

Arendt and Prüss [8, Theorem 5.1] have shown, under certain spectral conditions on $a$ and $A$, that $T(t) \stackrel{\mathrm{s}}{\rightarrow} P$ as $t \rightarrow \infty$, where $P=\lim _{\lambda \downarrow 0} \lambda \int_{0}^{\infty} e^{-\lambda t} T(t) d t$. For a precise formulation of this result, the reader is referred to [8]. The method of proof is similar in structure to [5], but the details are more complicated.

In the case when $a(t)=1$ for all $t$, then $\left\{T(t): t \in \mathbb{R}_{+}\right\}$is a bounded $C_{0^{-}}$ semigroup with generator $A$, and the result reduces to the fact that if $\sigma(A) \cap i \mathbb{R}$ is countable, $P \sigma\left(A^{*}\right) \cap i \mathbb{R} \subseteq\{0\}$ and Ker $A$ separates Ker $A^{*}$, then $T(t) \stackrel{\mathrm{s}}{\rightarrow} P$ as $t \rightarrow \infty$ (see Section 4.1). Other examples and applications to viscoelasticity may be found in [8].

4.5. Integrated semigroups [15]. Let $A$ be a closed linear operator in $X$ with domain $D(A)$ (not necessarily dense in $X$ ). A (once) integrated semigroup with generator $A$ is a strongly continuous family $S=\left\{S(t): t \in \mathbb{R}_{+}\right\}$of bounded linear operators on $X$ such that, for some $\omega>0,(\omega, \infty) \subseteq \varrho(A)$ and

$$
(\lambda I-A)^{-1}=\lambda \int_{0}^{\infty} e^{-\lambda t} S(t) d t \quad(\lambda>\omega) .
$$

Because of their relevance to the Cauchy problem, integrated semigroups have been much studied and generalised, since their introduction by Arendt [4].

Let $\left\{T(t): t \in \mathbb{R}_{+}\right\}$be a $C_{0}$-semigroup on $X$ with generator $A$, and put $S(t)=$ $\int_{0}^{t} T(s) d s$. Then $S$ is an integrated semigroup with generator $A$. If $0 \in \varrho(A)$, then $S(t)=(T(t)-I) A^{-1}$. Thus, the following result of El Mennaoui [15] is formally a generalisation of Theorem 3.1. However, the proof given in [15] uses Theorem 3.1 and an interpolation result for integrated semigroups, due to Arendt, Neubrander and Schlotterbeck [7]. We write $R \sigma(A)$ for the set of all $\lambda \in \mathbb{C}$ such that the range of $\lambda I-A$ is not dense in $X$, so $R \sigma(A)=P \sigma\left(A^{*}\right)$ if $A$ is densely defined. 
TheOREM $4.5\left[15\right.$, Corollaire 5.4]. Let $\left\{S(t): t \in \mathbb{R}_{+}\right\}$be an integrated semigroup with generator $A$, satisfying a Lipschitz condition: $\|S(t)-S(s)\| \leq K|t-s|$ $\left(s, t \in \mathbb{R}_{+}\right)$. Suppose that $\sigma(A) \cap i \mathbb{R}$ is countable, $R \sigma(A) \cap i \mathbb{R}$ is empty, and $0 \in \varrho(A)$. Then $S(t) \stackrel{\mathrm{s}}{\rightarrow}-A^{-1}$ as $t \rightarrow \infty$.

4.6. A converse result [31], [21]. Let $T$ be a power-bounded linear operator on a superreflexive space $X$. For each free ultrafilter $\mathcal{U}$ on $\mathbb{Z}_{+}$, let $X_{\mathcal{U}}$ be the associated ultrapower, and $T_{\mathcal{U}}$ be the operator on $X_{\mathcal{U}}$ induced by $T$. Then $X_{\mathcal{U}}$ is reflexive, $T_{\mathcal{U}}$ is power-bounded, and $\sigma\left(T_{\mathcal{U}}\right)=\sigma(T)$. Thus if $\sigma(T) \cap \Gamma$ is countable, then $T_{\mathcal{U}}$ is almost periodic by Theorem 4.3. Nagel and Räbiger [31] have proved the following converse result.

Theorem 4.6 [31, Theorem 3.7]. Let $T$ be a power-bounded operator on a superreflexive space $X$, and suppose that $T_{\mathcal{U}}$ is almost periodic for every ultrafilter $\mathcal{U}$ on $\mathbb{Z}_{+}$. Then $\sigma(T) \cap \Gamma$ is countable.

The argument in [31] uses the elementary fact that $\sigma(T) \cap \Gamma \subseteq P \sigma\left(T_{\mathcal{U}}\right)$. Assuming that $\sigma(T) \cap \Gamma$ is uncountable enables one to construct a non-atomic probability measure $\mu$ on $\sigma(T) \cap \Gamma$, a $T_{\mathcal{U}}$-invariant subspace $Y$ of $X_{\mathcal{U}}$ for some $\mathcal{U}$, and an isomorphism of $Y$ onto a Banach function lattice $W$ with $L^{\infty}(\mu) \subseteq W \subseteq$ $L^{1}(\mu)$ carrying $T_{\mathcal{U}} \mid Y$ onto the standard multiplier on $W$ (see Example $2.4(2)$ ). Since the multiplier is not almost periodic, this provides a contradiction. The construction in [31] uses a variety of results from the theory of Banach spaces and Banach lattices.

There is an analogous result for $C_{0}$-semigroups [21].

5. General semigroups. Let $S$ be a $\sigma$-compact subsemigroup of a locally compact abelian group $G$, with non-empty interior $S^{\circ}$ and with $S-S=G$. Let $S^{*}$ (respectively, $S_{\mathrm{u}}^{*}$ ) be the space of non-zero continuous bounded homomorphisms of $S$ into $\mathbb{C}$ (respectively, into $\Gamma$ ). Every character in the dual group $\widehat{G}$ restricts to a member of $S_{\mathrm{u}}^{*}$, and this enables us to identify $S_{\mathrm{u}}^{*}$ with $\widehat{G}$.

We consider $S$ with the restriction of Haar measure on $G$. For $f$ in $L^{1}(S)$ and $\chi$ in $S^{*}$, let

$$
\widehat{f}(\chi)=\int_{S} f(t) \chi(t) d t .
$$

We assume that the functions $\widehat{f}\left(f \in L^{1}(S)\right)$ separate the points of $S^{*}$ from each other and from 0. For example, this is satisfied if $S^{\circ}$ is dense in $S$.

We consider also a bounded representation $T$ of $S$ on $X$, so that $T$ is a strongly continuous map of $S$ into $\mathcal{B}(X)$ such that $T(s+t)=T(s) T(t)$ and $\sup _{t \in S}\|T(t)\|$ $<\infty$. For $f$ in $L^{1}(S)$, let

$$
\widehat{f}(T)=\int_{S} f(t) T(t) d t
$$


The spectrum $\operatorname{Sp}(T)$ of $T$ is the set of all $\chi$ in $S^{*}$ such that $|\widehat{f}(\chi)| \leq\|\widehat{f}(T)\|$ for all $f$ in $L^{1}(S)$. The unitary spectrum $\operatorname{Sp}_{\mathrm{u}}(T)$ is $\operatorname{Sp}(T) \cap S_{\mathrm{u}}^{*}$. The spectrum can naturally be identified with the Gelfand spectrum of the commutative Banach algebra $\mathcal{A}_{T}=\left\{\widehat{f}(T): f \in L^{1}(S)\right\}-[13$, Proposition 2.4].

The most natural examples are the multi-parameter semigroups, when $S=$ $\mathbb{Z}_{+}^{m}\left(G=\mathbb{Z}^{m}\right)$ or $S=\mathbb{R}_{+}^{m}\left(G=\mathbb{R}^{m}\right)$. When $S=\mathbb{Z}_{+}^{m}$, points $\chi$ in $S^{*}$ may be identified with points $z=\left(z_{1}, \ldots, z_{m}\right)$ in $D^{m}$, and bounded representations $T$ with $m$-tuples $\left(T_{1}, \ldots, T_{m}\right)$ of commuting power-bounded operators:

$$
\begin{aligned}
& \chi(t)=z_{1}^{n_{1}} \ldots z_{m}^{n_{m}} \\
& T(t)=T_{1}^{n_{1}} \ldots T_{m}^{n_{m}}
\end{aligned} \quad\left(t=\left(n_{1}, \ldots, n_{m}\right) \in \mathbb{Z}_{+}^{m}\right) .
$$

It is easy to see that $\operatorname{Sp}(T)$ is polynomially convex, in the sense of several complex variables.

When $S=\mathbb{R}_{+}^{m}$, points $\chi$ in $S^{*}$ may be identified with points $z$ in $\mathbb{C}_{-}^{m}$, and bounded representations $T$ with $m$-tuples $\left(T_{1}, \ldots, T_{m}\right)$ of commuting bounded $C_{0}$-semigroups:

$$
\begin{aligned}
& \chi(t)=\exp \left(t_{1} z_{1}+\ldots+t_{m} z_{m}\right) \\
& T(t)=T_{1}\left(t_{1}\right) \ldots T_{m}\left(t_{m}\right)
\end{aligned} \quad\left(t=\left(t_{1}, \ldots, t_{m}\right) \in \mathbb{R}_{+}^{m}\right) .
$$

Consider the case when $S=\mathbb{Z}_{+}$. The definition of $\operatorname{Sp}(T)$ as above is only partially consistent with the operator-theoretic definition of $\sigma(T)$. In fact, $\operatorname{Sp}(T)$ is the polynomially convex hull of $\sigma(T)$, so $\mathbb{C} \backslash \operatorname{Sp}(T)$ is the unbounded component of $\varrho(T)$. Similarly, when $S=\mathbb{R}_{+}, \operatorname{Sp}(T)$ is an appropriate hull of $\sigma(A)$, where $A$ is the generator. Nevertheless, our definition does not create an unreasonably large spectrum, because we are essentially interested only in the unitary spectrum, which the following proposition shows to be as small as possible.

Proposition 5.1 [13, Proposition 2.2]. Let $T$ be a bounded representation of $S$ on $X$, and $\chi \in S_{\mathrm{u}}^{*}$. Then $\chi \in \mathrm{Sp}_{\mathrm{u}}(T)$ if and only if $\chi$ is an approximate eigenvalue of $T$, that is, there is a sequence $\left(x_{n}\right)$ in $X$ with $\left\|x_{n}\right\|=1$ such that $\left\|T(t) x_{n}-\chi(t) x_{n}\right\| \rightarrow 0$ as $n \rightarrow \infty$ (uniformly for $t$ in compact subsets of $S$ ).

We have seen in Proposition 2.6 and the proof of Theorem 3.1 that the question of convergence to zero of a bounded one-parameter semigroup of operators is closely related to spectral properties of isometric semigroups. We shall use this approach to general semigroups later in this section, so we establish first some facts about the isometric case. In the first proposition, we identify $\operatorname{Sp}(T)$ with the Gelfand spectrum of $\mathcal{A}_{T}$. If $\mathcal{A}_{T}$ is non-unital, we further identify $\operatorname{Sp}(T)$ with a subset of the Gelfand spectrum of $\mathcal{A}_{T}+\mathbb{C} I$, and we take the Shilov boundary of $\mathcal{A}_{T}$ to be the part of the Shilov boundary of $\mathcal{A}_{T}+\mathbb{C} I$ which lies in $\operatorname{Sp}(T)$.

Proposition 5.2 [13], [11]. Let $T$ be a representation of $S$ by isometries on $X$. Then $\operatorname{Sp}_{\mathrm{u}}(T)$ is the Shilov boundary of the commutative Banach algebra $\mathcal{A}_{T}$. Hence, any isolated point of $\operatorname{Sp}_{\mathrm{u}}(T)$ is an eigenvalue of $T$. 
The proof of Proposition 5.2 depends on two facts from the theory of commutative Banach algebras. Firstly, a theorem of Żelazko [40] shows that any point in the Shilov boundary of $\mathcal{A}_{T}$ is an approximate eigenvalue for $T$; secondly, any isolated point of the Shilov boundary is isolated in the Gelfand spectrum [39, p. 55].

The following dilation theorem of Greenfield reduces many aspects of the study of isometric semigroups to that of isometric groups. The point spectrum $P \sigma\left(T^{*}\right)$ of $T^{*}$ is the set of all $\chi$ in $S^{*}$ for which there exist non-zero $\phi$ in $X^{*}$ such that $T(t)^{*} \phi=\chi(t) \phi$ for all $t$, and the unitary point spectrum of $T^{*}$ is $P \sigma_{\mathrm{u}}\left(T^{*}\right)=P \sigma\left(T^{*}\right) \cap S_{\mathrm{u}}^{*}$.

Theorem 5.3 [19]. Let $T$ be a representation of $S$ by isometries on $X$. There is a Banach space $Z$ containing $X$ (isometrically) and a representation $U$ of $G$ by (invertible) isometries on $Z$, such that

(1) for each $t$ in $S, X$ is $U(t)$-invariant and $T(t)=U(t) \mid X$,

(2) $Z=\{U(t) x: t \in G, x \in X\}^{-}$,

(3) $\operatorname{Sp}(U)=\operatorname{Sp}_{\mathrm{u}}(T), P \sigma\left(U^{*}\right)=P \sigma_{\mathrm{u}}\left(T^{*}\right)$.

Proof. A complete bounded trajectory for $T^{*}$ is a bounded family $\phi=\left\{\phi_{t}\right.$ : $t \in G\}$ in $X^{*}$ such that $T(s)^{*} \phi_{t}=\phi_{s+t}(s \in S, t \in G)$. Let $Y$ be the Banach space of all complete bounded trajectories for $T^{*}$, with $\|\phi\|=\sup _{t \in G}\left\|\phi_{t}\right\|$. For $t$ in $G$, define an isometry $L(t)$ on $Y$ by $(L(t) \phi)_{s}=\phi_{s+t}$.

For $x$ in $X$, define $\widehat{x}$ in $Y^{*}$ by

$$
\widehat{x}(\phi)=\phi_{0}(x) .
$$

Let $\psi \in X^{*}$. Since $T$ is isometric, it is straightforward to construct a complete bounded trajectory $\phi$ for $T^{*}$ with $\|\phi\|=\|\psi\|$ and $\phi_{0}=\psi$. It follows that $\|\widehat{x}\|=$ $\|x\|$. Define

$$
Z=\left\{L(t)^{*} \widehat{x}: t \in G, x \in X\right\}^{-}, \quad U(t)=L(t)^{*} \mid Z .
$$

If we identify $X$ with $\{\widehat{x}: x \in X\}$, then all the required properties may be verified.

Both parts of the following corollary are easily deduced from Theorem 5.3 and the corresponding results for isometric representations of groups [34, 8.1]. For norm-continuous representations, the first part can be proved by ultrapower techniques [27]. For strongly continuous representations, it was originally proved in [13] by means of a Banach algebra construction of Arens [9].

Corollary 5.4 [13, Corollary 3.3], [11], [19]. Let $T$ be a representation of $S$ by isometries on $X$.

(1) If $X \neq\{0\}$, then $\operatorname{Sp}_{\mathrm{u}}(T)$ is non-empty.

(2) If $\mathrm{Sp}_{\mathrm{u}}(T)$ is compact, then $T$ is norm-continuous.

We also saw in Remark 2.7.1 and Proposition 3.2 that if the spectrum of a one-parameter isometric representation is not as large as possible, then the 
operators are invertible. The following is a general result in this direction. More detailed results for multi-parameter semigroups will be given in Section 6 .

TheOREM 5.5 [11], [19]. Let $T$ be a representation of $S$ by isometries on $X$, and suppose that $\operatorname{Sp}_{\mathrm{u}}(T)$ is countable. Then each $T(t)$ is invertible.

Proof. Let $Y=\bigcap_{t \in S} \operatorname{Ran} T(t), \widetilde{X}=X / Y$, and $\widetilde{T}$ be the representation of $S$ by isometries on $\widetilde{X}$ induced by $T$. It can be verified that $\operatorname{Sp}_{\mathrm{u}}(\widetilde{T}) \subseteq \operatorname{Sp}_{\mathrm{u}}(T)$. If $\widetilde{X} \neq\{0\}$, then, by Corollary $5.4(1)$ and the countability assumption, $\operatorname{Sp}_{\mathrm{u}}(\widetilde{T})$ has an isolated point $\chi$. By Proposition 5.2, $\chi$ is an eigenvalue of $\widetilde{T}$. However, the definition of $\widetilde{X}$ ensures that $\widetilde{T}$ has no eigenvalues. This contradiction implies that $Y=X$, so each $T(t)$ is invertible.

The proof of Theorem 5.5 in [19] replaces the use of Banach algebra techniques (within Proposition 5.2) by a precise analysis of the constructions used in Theorem 5.3.

In the light of Proposition 5.1, parts (1) and (2) of Theorem 5.6 below are generalisations of Theorems 2.5 and 3.1, and of Theorems 2.1 and 3.3. For $T$ norm-continuous, part (1) was first proved by Lyubich and Phóng [30] and part (2) by Phóng [35] (for $S=\mathbb{Z}_{+}^{m}$, part (2) had already been proved in [23]). For $T$ strongly continuous, both parts were first proved in [13], and a simpler proof has been obtained by Greenfield [19].

For multi-parameter semigroups, it is sometimes possible to reach the conclusions of Theorem 5.6 by applying Theorem 2.1, 2.5, 3.1, or 3.3 to a suitably chosen one-parameter subsemigroup. However, an example was given in [13] where $S=\mathbb{R}_{+}^{2}$ and the conditions of Theorem 5.6(1) are satisfied, but the spectrum of every one-parameter subsemigroup is $\mathbb{C}_{-}$, so Theorem 3.1 is not applicable.

The semigroup $S$ is a directed set in the ordering defined by: $t \succeq s \Leftrightarrow t-s \in S$. A function $f$ in $L^{1}(S)$ is of spectral synthesis for a closed subset $E$ of $\widehat{G}$ if there is a sequence $\left(g_{n}\right)$ in $L^{1}(G)$ such that $\widehat{g}_{n}=0$ in a neighbourhood of $E$ and $\left\|f-g_{n}\right\|_{1} \rightarrow 0$ as $n \rightarrow \infty$.

Theorem 5.6 [23, Theorem 8], [30], [35, Theorem 4.2], [13, Theorem 4.2], [11]. Let $T$ be a bounded representation of $S$.

(1) If $\operatorname{Sp}_{\mathrm{u}}(T)$ is countable and $P \sigma_{\mathrm{u}}\left(T^{*}\right)$ is empty, then $T(t) \stackrel{\mathrm{s}}{\rightarrow} 0$ as $t \rightarrow \infty$ (through $S$ ).

(2) If $f$ in $L^{1}(S)$ is of spectral synthesis for $\operatorname{Sp}_{\mathrm{u}}(T)$, then $\|T(t) \widehat{f}(T)\| \rightarrow 0$ as $t \rightarrow \infty$ (through $S)$.

Proof. By a construction analogous to Proposition 2.6, there is a Banach space $E$, a bounded linear map $Q: X \rightarrow E$ with dense range, and a representation $U$ of $S$ by isometries on $E$ such that $\operatorname{Sp}(U) \subseteq \operatorname{Sp}(T), P \sigma\left(U^{*}\right) \subseteq P \sigma\left(T^{*}\right), Q T(t)=$ $U(t) Q$, and $\|T(t) x\| \rightarrow 0$ for all $x$ in $\operatorname{Ker} Q$. By Theorem 5.3, there is a Banach space $Z$ containing $E$, and a representation $V$ of $G$ by isometries on $Z$ such that 
$U(t)=V(t) \mid E(t \in S), \operatorname{Sp}(V)=\operatorname{Sp}_{\mathrm{u}}(U) \subseteq \operatorname{Sp}_{\mathrm{u}}(T)$, and $P \sigma\left(V^{*}\right)=P \sigma_{\mathrm{u}}\left(U^{*}\right) \subseteq$ $P \sigma_{\mathrm{u}}\left(T^{*}\right)$.

(1) Suppose that $T(t)$ does not converge strongly to 0 . Then $E \neq\{0\}$, so $Z \neq\{0\}$ (in fact, $Z=E$ by Theorem 5.5). Since $\operatorname{Sp}(V)$ is countable, it has an isolated point $\chi$. Now $\chi$ is an eigenvalue of $V$, so $\chi \in P \sigma\left(V^{*}\right) \subseteq P \sigma_{\mathrm{u}}\left(T^{*}\right)$, a contradiction.

(2) If $f$ is of spectral synthesis for $\operatorname{Sp}_{\mathrm{u}}(T)$ and hence for $\operatorname{Sp}(V)$, then $\widehat{f}(V)=0$, by the theory of isometric representations of groups. It follows routinely that $T(t) \widehat{f}(T) \stackrel{\mathrm{s}}{\rightarrow} 0$. To establish norm-convergence, one uses the devices outlined in Remark 2.7.2 and Remark 3.4.2.

There is a version of Theorem 5.6 for almost periodic semigroups, analogous to Theorem 4.3 [13, Theorem 5.1].

6. Multi-parameter semigroups of isometries. We have seen in Theorem 5.5 that if $T$ is an isometric representation of a general semigroup $S$ and $\operatorname{Sp}_{\mathrm{u}}(T)$ is countable, then each $T(t)$ is invertible. If $T$ is an isometric representation of $\mathbb{R}_{+}^{m}$ and $\mathrm{Sp}_{\mathrm{u}}(T)$ is compact, then it follows from Corollary 5.4 that each $T(t)$ is invertible. Here, we give some further results of this type for multi-parameter semigroups of isometries.

We saw in Remark 2.7.1 and Proposition 3.2 that for one-parameter semigroups of isometries, the assumption that $\operatorname{Sp}(T) \neq S^{*}$ is sufficient to ensure invertibility. However, for multi-parameter semigroups, this assumption is much too weak. Let $T_{0}: X \rightarrow X$ be a non-invertible isometry, and define a representation $T$ of $\mathbb{Z}_{+}^{2}$ by $T(m, n)=T^{m+n}$. Then $\operatorname{Sp}(T)=\{(z, z): z \in D\}$.

ExAmple 6.1. Let $E$ be a closed subset of $\widehat{G}=S_{\mathrm{u}}^{*}$, and let $J_{E}$ be the ideal of all functions in $L^{1}(G)$ which are of spectral synthesis with respect to $E$. Let $X_{E}$ be the closure of $\left(L^{1}(S)+J_{E}\right) / J_{E}$ in $L^{1}(G) / J_{E}$, and $T_{E}$ be the isometric representation of $S$ on $X_{E}$ induced by translations:

$$
T_{E}(s)\left(f+J_{E}\right)=f_{s}+J_{E},
$$

where $f_{s}(t)=f(t-s)$. Then $\operatorname{Sp}_{\mathrm{u}}\left(T_{E}\right)=E$ [11]. Moreover, the dilation of $T_{E}$ given by Theorem 5.3 is (up to isometric isomorphism) the representation of $G$ on $L^{1}(G) / J_{E}$ induced by translations. In particular, $T_{E}$ is invertible if and only if $L^{1}(S)+J_{E}$ is dense in $L^{1}(G)$.

Proposition 6.2 [11]. Let $E$ be a closed subset of $\widehat{G}$, and $T_{E}$ be as in Example 6.1. The following are equivalent:

(1) Every representation $T$ of $S$ by isometries with $\operatorname{Sp}_{\mathrm{u}}(T)=E$ is invertible;

(2) $T_{E}$ is invertible;

(3) $L^{1}(S)+J_{E}$ is dense in $L^{1}(G)$.

If $S$ is discrete, these conditions are equivalent to: 
(4) $\operatorname{Sp}(T) \subseteq \widehat{G}$ for some (or all) representation $T$ of $S$ by isometries with $\operatorname{Sp}_{\mathrm{u}}(T)=E$.

For discrete multi-parameter semigroups of isometries, the question of automatic invertibility reduces to the question of polynomial convexity (in the sense of several complex variables) of the compact subset $\operatorname{Sp}_{u}(T)$ of the $m$-torus $\Gamma^{m}$. This question has been studied by Stolzenberg [37] and Alexander [1]. For a compact subset $E$ of $\mathbb{C}^{m}, \widehat{E}$ will denote the polynomially convex hull of $E$; $E$ is said to be simply-coconnected if every continuous function $f: E \rightarrow \mathbb{C} \backslash\{0\}$ has a continuous logarithm, or equivalently if the cohomology group $H^{1}(E, \mathbb{Z})$ vanishes [38, Theorem 10.1]. Any contractible set is simply-coconnected.

THEOREM 6.3 [11]. Let $T=\left(T_{1}, \ldots, T_{m}\right)$ be a representation of $\mathbb{Z}_{+}^{m}$ by isometries on $X$.

(1) $\operatorname{Sp}(T)=\operatorname{Sp}_{\mathrm{u}}(T)^{\wedge}$.

(2) If $\mathrm{Sp}_{\mathrm{u}}(T)$ is polynomially convex, then each $T_{j}$ is invertible.

(3) If $\operatorname{Sp}_{\mathrm{u}}(T)$ is contained in a simply coconnected subset of $\Gamma^{m}$, or if $\operatorname{Sp}_{\mathrm{u}}(T)$ is contained in a Jordan arc, or if $\mathrm{Sp}_{\mathrm{u}}(T)$ is totally disconnected, then each $T_{j}$ is invertible.

For continuous multi-parameter semigroups, the question of automatic invertibility is also related to polynomial convexity, but in an indirect fashion. Let $\theta: \mathbb{C}_{-} \rightarrow D$ be the Möbius transform defined by $\theta(z)=\frac{z+1}{z-1}$, and let $\theta_{m}: \mathbb{C}_{-}^{m} \rightarrow D^{m}$ be the $m$-fold copy of $\theta$. Let $K=\left\{z \in D^{m}: z_{j}=1\right.$ for some $j$ \}, so $\theta_{m}$ maps $i \mathbb{R}^{m}$ onto $\Gamma^{m} \backslash K$.

THEOREM 6.4 [11]. Let $T$ be a representation of $\mathbb{R}_{+}^{m}$ by isometries on $X$, and let $E=\overline{\theta_{m}\left(\mathrm{Sp}_{\mathrm{u}}(T)\right)}$.

(1) $\operatorname{Sp}(T)=\theta_{m}^{-1}(\widehat{E} \backslash K)$.

(2) If $\widehat{E} \subseteq E \cup K$, then $\operatorname{Sp}_{\mathrm{u}}(T)=\operatorname{Sp}(T)$.

(3) If $\mathrm{Sp}_{\mathrm{u}}(T)$ is the union of its relatively open compact subsets, then each $T(t)$ is invertible.

(4) If $E \cap K$ is polynomially convex and $\mathrm{Sp}_{\mathrm{u}}(T)$ is totally disconnected, then each $T(t)$ is invertible.

\section{References}

[1] H. Alexander, On a problem of Stolzenberg in polynomial convexity, Illinois J. Math. 22 (1978), 149-160.

[2] G. R. Allan, A. G. O'Farrell and T. J. Ransford, A Tauberian theorem arising in operator theory, Bull. London Math. Soc. 19 (1987), 537-545.

[3] G. R. Allan and T. J. Ransford, Power-dominated elements in a Banach algebra, Studia Math. 94 (1989), 63-79.

[4] W. Arendt, Resolvent positive operators, Proc. London Math. Soc. 54 (1987), 321-349. 
[5] W. Arendt and C. J. K. Batty, Tauberian theorems and stability of one-parameter semigroups, Trans. Amer. Math. Soc. 306 (1988), 837-852.

[6] - - - A complex Tauberian theorem and mean ergodic semigroups, preprint.

[7] W. Arendt, F. Neubrander and U. Schlotterbeck, Interpolation of semigroups and integrated semigroups, Semesterbericht Funktionalanalysis Tübingen 15 (1988/89), 1-14.

[8] W. Arendt and J. Prüss, Vector-valued Tauberian theorems and asymptotic behavior of linear Volterra equations, SIAM J. Appl. Math. 23 (1992), 412-448.

[9] R. Arens, Inverse-producing extensions of normed algebras, Trans. Amer. Math. Soc. 88 (1958), 536-548.

[10] C. J. K. Batty, Tauberian theorems for the Laplace-Stieltjes transform, ibid. 322 (1990), $783-804$.

[11] C. J. K. Batty and D. A. Greenfield, On the invertibility of isometric semigroup representations, preprint.

[12] C. J. K. Batty and Vũ Quôc Phóng, Stability of individual elements under oneparameter semigroups, Trans. Amer. Math. Soc. 322 (1990), 805-818.

[13] - - - Stability of strongly continuous representations of abelian semigroups, Math. Z 209 (1992), 75-88.

[14] E. B. Davies, One-Parameter Semigroups, Academic Press, London, 1980.

[15] O. El Mennaoui, Comportement asymptotique des semi-groupes intégrés, J. Comput. Appl. Math., to appear.

[16] J. Esterle, E. Strouse and F. Zouakia, Theorems of Katznelson-Tzafriri type for contractions, J. Funct. Anal. 94 (1990), 273-287.

[17] - - - - Stabilité asymptotique de certains semigroupes d'opérateurs, J. Operator Theory, to appear.

[18] I. Gelfand, Zur Theorie der Charaktere der abelschen topologischen Gruppen, Mat. Sb. 9 (51) (1941), 49-50.

[19] D. A. Greenfield, D.Phil. thesis, Oxford, in preparation.

[20] E. Hille and R. S. Phillips, Functional Analysis and Semi-groups, Amer. Math. Soc. Providence, 1957.

[21] S. Huang and F. Räbiger, Superstable $C_{0}$-semigroups on Banach spaces, preprint.

[22] A. E. Ingham, On Wiener's method in Tauberian theorems, Proc. London Math. Soc. 38 (1935), 458-480.

[23] Y. Katznelson and L. Tzafriri, On power bounded operators, J. Funct. Anal. 68 (1986), 313-328.

[24] J. Korevaar, On Newman's quick way to the prime number theorem, Math. Intelligencer 4 (1982), 108-115.

[25] U. Krengel, Ergodic Theorems, de Gruyter, Berlin, 1985.

[26] Yu. I. Lyubich, On the spectrum of a representation of an abelian topological group, Dokl. Akad. Nauk SSSR 200 (1971), 777-780 (in Russian); English transl.: Soviet Math. Dokl. 12 (1971), 1482-1486.

[27] - Introduction to the Theory of Banach Representations of Groups, Birkhäuser, Basel, 1988.

[28] Yu. I. Lyubich and Vũ Quôc Phóng, Asymptotic stability of linear differential equations on Banach spaces, Studia Math. 88 (1988), 37-42.

[29] - - - A spectral criterion for almost periodicity of one-parameter semigroups, Teor. Funktsiu Funktsional. Anal. i Prilozhen. 47 (1987), 36-41 (in Russian).

[30] - - - A spectral criterion for almost periodicity of representations of abelian semigroups, ibid. 51 (1987) (in Russian).

[31] R. Nagel and F. Räbiger, Superstable operators on Banach spaces, Israel J. Math. 81 (1993), 213-226. 
[32] B. Sz.-Nagy and C. Foiaş, Harmonic Analysis of Operators on Hilbert Space, NorthHolland, Amsterdam, 1970.

[33] D. J. New man, Simple analytic proof of the prime number theorem, Amer. Math. Monthly 87 (1980), 693-696.

[34] G. K. Pedersen, $C^{*}$-Algebras and Their Automorphism Groups, Academic Press, London, 1979.

[35] Vũ Quôc Phóng, Theorems of Katznelson-Tzafriri type for semigroups of operators, J. Funct. Anal. 103 (1992), 74-84.

[36] G. M. Sklyar and V. Ya. Shirman, On the asymptotic stability of a linear differential equation in a Banach space, Teor. Funktsiü Funktsional. Anal. i Prilozhen. 37 (1982), 127-132 (in Russian).

[37] G. Stolzenberg, Polynomially and rationally convex sets, Acta Math. 109 (1963), 259289.

[38] E. L. Stout, The Theory of Uniform Algebras, Bogden \& Quigley, Tarrytown-on-Hudson, 1971.

[39] J. Wermer, Banach Algebras and Several Complex Variables, Springer, New York, 1976.

[40] W. Żelazko, On a certain class of non-removable ideals in Banach algebras, Studia Math. 44 (1972), 87-92.

Added in proof. Since this article was completed, it has come to the author's attention that Theorem 5.3 was obtained by R. G. Douglas (Bull. London Math. Soc. 1 (1969), 157-159). 